\title{
Frequency Reconfigurable Vivaldi Antenna with Switched Resonators for Wireless Applications
}

\author{
Rabiaa Herzi ${ }^{1}$, Ali Gharsallah ${ }^{4}$ \\ Unit of Research CSEHF Faculty of Sciences of Tunis \\ El Manar, Tunis 2092, Tunisia
}

\author{
Mohamed-Ali Boujemaa ${ }^{2}$ Fethi Choubani ${ }^{3}$ \\ INNOVCOM Laboratory, SUPCOM, University of \\ Carthage, Tunis, Tunisia
}

\begin{abstract}
In this paper, a frequency reconfigurable Vivaldi antenna with switched slot ring resonators is presented. The principle of the method to reconfigure the Vivaldi antenna is based on the perturbation of the surface currents distribution. Switched ring resonators that act as a bandpass filter are printed in specific positions on the antenna metallization. This structure has the ability to reconfigurate between wideband mode and four narrow-band modes which cover significant wireless applications. Combination of the bandpass filters and tapered slot antenna characteristics achieve an agile antenna capable to operate in UWB mode from 2 to $8 \mathrm{GHz}$ and to generate multinarrow bands at $3.5 \mathrm{GHz}, 4 \mathrm{GHz}, 5.2 \mathrm{GHz}, 5.5 \mathrm{GHz}, 5.8 \mathrm{GHz}$ and 6.5 GHz. The measurement and simulation results show good agreement. This antenna is an appropriate solution for wireless applications which require reconfigurable Wideband multinarrow bands antenna.
\end{abstract}

Keywords-Frequency reconfigurable; Vivaldi Antenna (VA); Ultra-Wideband (UWB); slot ring resonator; wireless applications

\section{INTRODUCTION}

Research in antenna development has attracted many researches to satisfy the requirements of modern wireless applications such as developing active, compact and miniaturized antennas that can group many services [1-3].

Developing of radio systems for multimode terminal applications which involve a combination of Wi-Fi, WLAN, Wimax, Bluetooth... is obligatory $[4,5]$. So, using systems with frequency reconfigurable operation and wideband spectrum sensing is a suitable solution due to its compactness, flexibility and its capability to operate over multiple bands [56].

The reconfigurable antenna is an antenna which has the capability to change dynamically its radiation characteristics such as its radiation patterns, frequency operations or its polarization [7-8]. Usually, the reconfigurability of antenna is achieved using PIN diodes, Varactors or MEMS to change its geometrical characteristics $[1,4]$.

Because of their benefits of flexibility, the capability to reduce interferences and compactness, frequency reconfigurable antenna is a better alternative which can be used in cognitive radio and multi-mode applications [9]. More precisely, they are many modern systems which have great numbers of antennas that are operated at different frequencies. Therefore, frequency reconfigurable antenna, which can support many functions at various frequencies bands and can significantly decrease the hardware cost and size, is required.
There are many types of frequency reconfigurable antennas such as switching between different narrow bands, wideband to notch band reconfiguration, wideband to narrowband switching [10-11].

Achieving an antenna which has the capacity of wideband to multi-narrow bands reconfiguration is very important and essential for several applications such as a cognitive radio that uses wideband sensing and multi-bands communications $[10$, $12,13]$.

Because of their better radiation performances as well as Ultra-wide bandwidth, elevated gain, and compact structure [14-15], Vivaldi antenna is the best selection to be used in wideband to multi-bands reconfiguration.

Developing of reconfigurable antenna between wideband and multiple narrow-bands has received considerable attention. In [16] and [17] two different switchable Vivaldi antennas have been investigated where several PIN diodes and capacitors are used which augment the complexity of the antenna design. In addition, the reconfigurability of both antennas has notably deteriorated the antenna gain which has decreased by about $2.5 \mathrm{dBi}$. A reconfigurable Vivaldi antenna was designed in [18], where the obtained agile narrow bands are close with inconstant radiation patterns over the operating frequency range. A wideband frequency agile patch antenna is proposed in [19]. The reconfigurability is achieved using Varactors which require high bias voltages. Very close frequency reconfigurable bands are attained from 1.47 to 1.84 $\mathrm{GHz}$.

In this paper, a frequency reconfigurable Vivaldi antenna for wireless applications that has the capacity to switch from an UWB of 2-8 GHz to essential narrow bands is proposed. By inserting of switchable slot ring resonators using PIN diodes, several narrow bands are obtained. In Section 2, details of the UWB antenna and the proposed antenna design are described. The principle of the method used to achieve a wide to narrow-bands reconfigurable Vivaldi antenna is demonstrated in Section 3. In Section 4, the simulated and measured results obtained throughout the switch modes are explained and discussed. Finally, Section 5 presents the conclusion of this paper.

\section{UWA AND RECONFIGURABLE ANTENNAS DESIGN}

The basic structure of the proposed antenna, which is shown in Fig. 1(a) is an UWB Vivaldi antenna that operates from 2 to $8 \mathrm{GHz}$. It consists of an exponentially tapered slot 
printed on an FR-4 substrate that has 4.7 of permittivity and $1.575 \mathrm{~mm}$ of thickness. It is fed by a microstrip line printed on the other side of the substrate. The dimension of the antenna is $\mathrm{L}=85 \mathrm{~mm}$ and $\mathrm{W}=70 \mathrm{~mm}$. The exponential edge of the aperture antenna is determined using next equations:

Where $\mathrm{f},\left(x_{1}, y_{1}\right)$ and $\left(x_{2}, y_{2}\right) Y= \pm\left(A e^{f x}+B\right)$

$A=\frac{y_{2}-y_{1}}{e^{f x_{2}}-e^{f x_{1}}}$

$B=\frac{y_{1} e^{f x_{2}}-y_{2} e^{f x_{1}}}{e^{f x_{2}-e^{f x_{1}}}}$

Are respectively the exponential factor, the peak point and the bottom point of the exponential edge.

Fig. 1(b) shows the wide-narrow bands agile antenna configuration. The Vivaldi antenna geometry is modified by inserting four slot resonators at specific positions in order to perturb the surface currents flow. Each resonator is formed by two symmetric ring slots connected by a rectangular gap. So, three pairs of ring slots are printed on the antenna metallization and coupled into the tapered slot through four pairs of gaps with $1 \mathrm{~mm}$ of width. The outer and the inner radius of the ring slots are respectively $5 \mathrm{~mm}$ and $3 \mathrm{~mm}$. Moreover, ten PIN diodes are inserted in the opening of the perturbing slots to switch the function of the different resonators and control the current distributions.

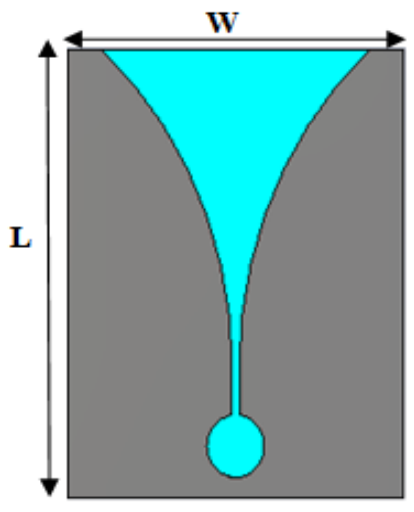

(a)

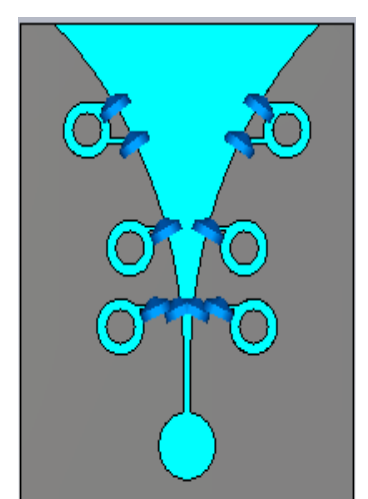

(b)
Fig. 1. Simple Vivaldi Antenna (a) and Reconfigurable Vivaldi Antenna with PIN Diodes (b).

\section{PRINCIPLE OF RECONFIGURATION OF THE VIVALDI ANTENNA}

The Ultra-Large Band that characterizes the Vivaldi antenna is the result of the tapered slot geometry that can be devised into two parts named the propagation and radiation parts. The propagation part guides the waves to the radiation area. Each level of the radiation part radiate at the corresponding frequency, where the width of the slot is about the half wavelength.

Generally, frequency reconfigurable Vivaldi antenna can be achieved by disturbing the surface currents flow which is attained by distorting the inner edges of the tapered slot. Moreover, inserting slot ring resonator can act as a filter which has the capability to pass a frequency band and block others. Single ring slot acts as a stop band filter, blocking the frequencies that correspond to a quarter of the wavelength $(\lambda / 4)$, while double serial ring slots act as pass band filter which pass frequencies corresponding to a half wavelength $(\lambda / 2)$. Then, the resonance current path length (D) the stop band and pass band filters can be expressed respectively as following:

$D_{S}=\frac{\lambda_{\mathrm{eff}}}{4} \approx(P+s)$

$D_{P}=\frac{\lambda_{\text {eff }}}{2} \approx(P+2 s)$

The resonance frequencies of the stop band and pass band filters can be determined as follows:

$F_{S}=\frac{c}{4 \sqrt{\varepsilon_{e f f}}(P+s)}$

$F_{P}=\frac{c}{2 \sqrt{\varepsilon_{e f f}}(P+2 s)}$

Where $\mathrm{c}, \varepsilon_{\text {eff }}, \mathrm{P}$ and $\mathrm{S}$ are, respectively, the velocity of light in the free space, the effective permittivity of the substrate, the perimeter of the ring slot which is equal to 31.4 $\mathrm{mm}$ and the width of the split.

Fig. 2 and Fig. 3 illustrate the structure and the response of a single and double rings slot resonator. It is clear that the single slot ring resonator products a stop band response around $2.14 \mathrm{GHz}$ while the double rings resonator offers a pass-band filter at $3.65 \mathrm{GHz}$.

The equivalent circuit of the double slot rings resonators which consists of two associated stop band filters is shown in Fig. 4. Two LC circuits are connected in series, where each LC circuit shows a stop band filter.

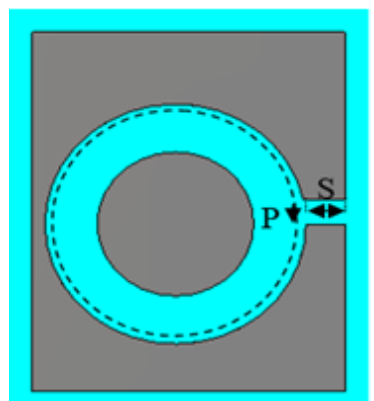

(a)

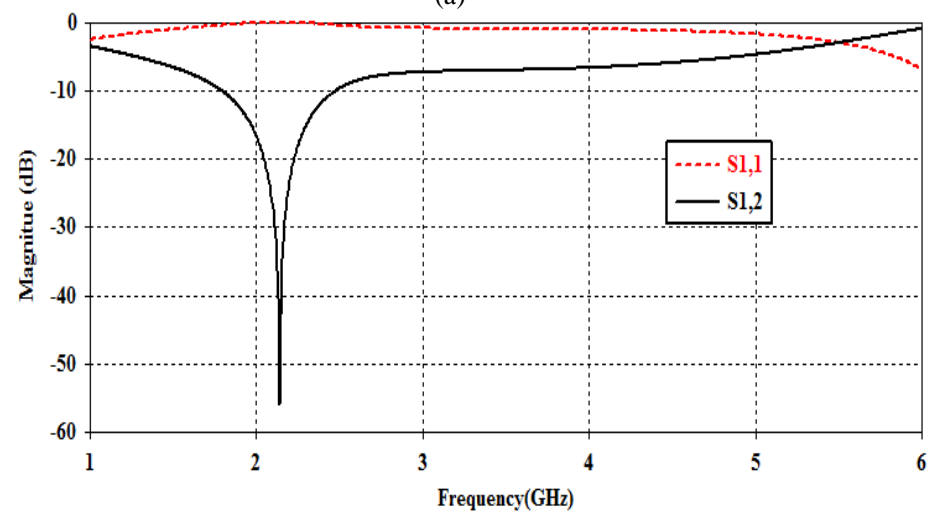

(b)

Fig. 2. Configuration of Single Ring Slot Resonator (a) and the SParameters Response (b). 


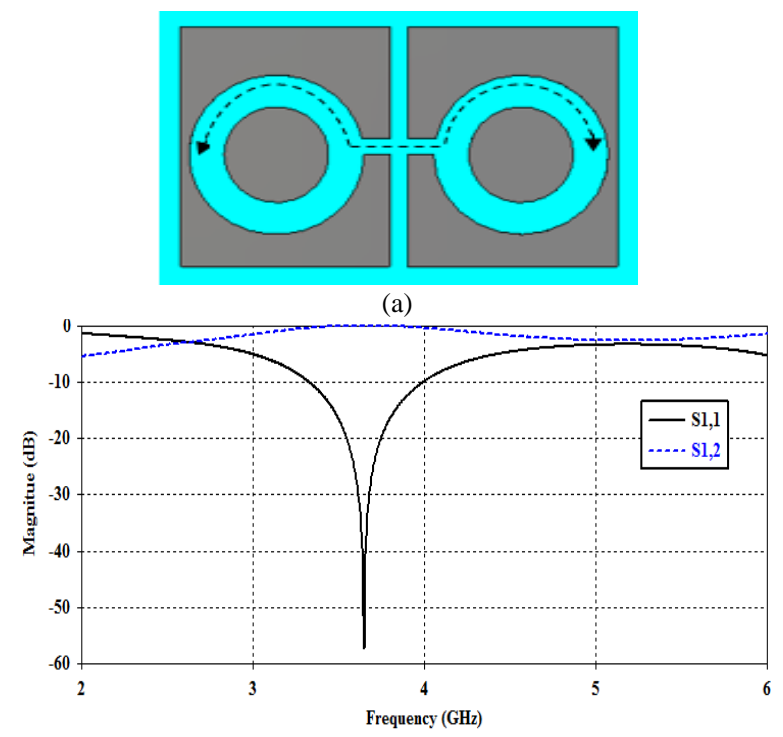

(b)

Fig. 3. Configuration of Double Rings Slot Resonator (a) and the SParameters Response (b).

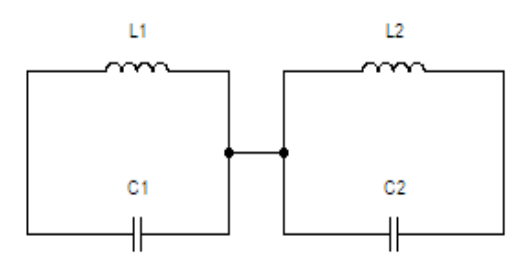

Fig. 4. Equivalent Circuit of the Double Rings Slot Resonator.

To investigate the effects of the slots ring resonator in the radiation characteristics of the Vivaldi antenna, a double rings slot resonator is inserted in the beginning of the radiation part. Fig. 5 presents the surface currents distribution of the Vivaldi antenna with and without printed resonator. It can be seen that the current before inserting perturbation follows the direction of the inner radiation edges of the antenna. On the other hand, the surface current is distorted by inserting of the ring slots; it is significantly reduced along the inner edges. So the major asset of this method resides in the capability to promote the disruption of the currents in the annular slots and stopped its repartition along the radiation edges situated after the disturbance. In addition, Fig. 6 compares the reflection coefficients of the simple and the modified antenna. It is clear that the impedance matching is distorted by inserting of the ring slots in the beginning of the radiation part, where the reflection coefficient is up to $-10 \mathrm{~dB}$ over the frequency range. So, up to the perturbation, the surface currents distribution along the edges of the radiation area is blocked.

As can be seen, inserting a ring slot resonator has two roles: to generate a band-pass response at the frequency corresponding to the half wavelength and to block the surface currents distribution which makes the area situated up to the perturbation in the off state. So, reconfigurable Vivaldi antenna can be achieved by varying the level $(\mathrm{Y})$ of the perturbation and the width of the resonant current path (D) that are the important parameters where the inserted resonator can operate as a filter which passes a range of frequencies and blocks others. In this way, four modes are investigated. Fig. 7 shows four proposed structures with different positions of the printed resonator. Fig. 8 compares the reflection coefficients of the different structures where four narrow-band modes are achieved.

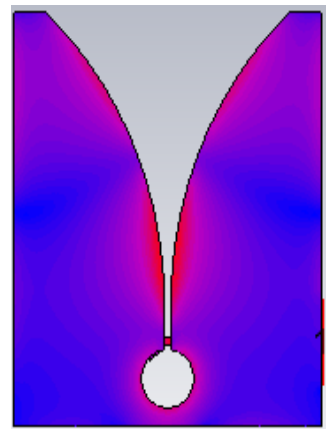

(a)

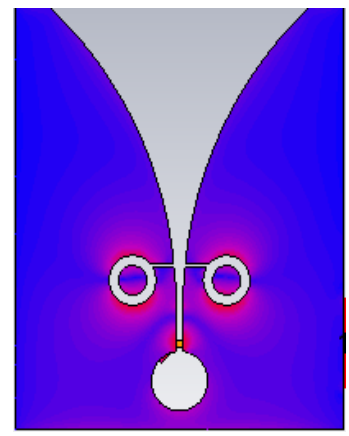

(b)
Fig. 5. Surface Currents Distribution of (a) the Simple Vivaldi Antenna and (b) Vivaldi Antenna with Annular Slots at $3.5 \mathrm{GHz}$.

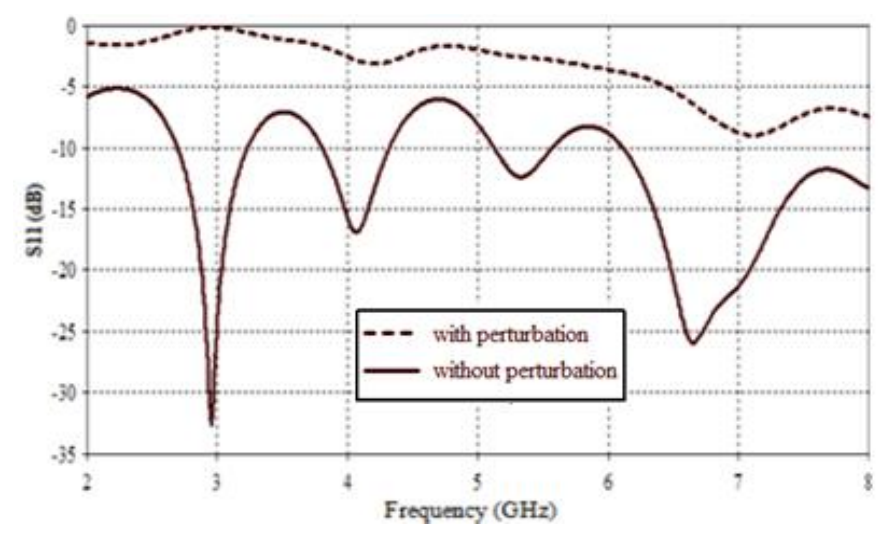

Fig. 6. Reflection Coefficients of the Antenna with and without Perturbation.

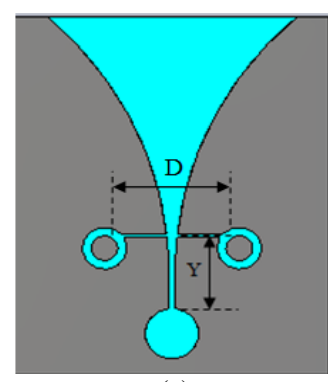

(a)

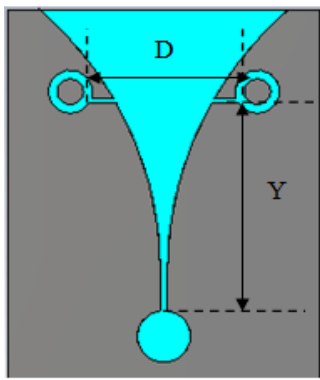

(c)

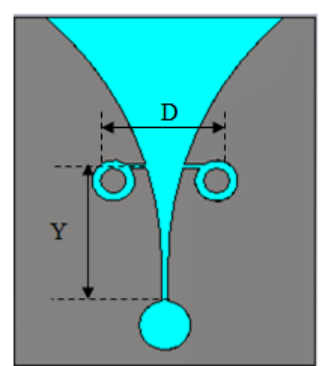

(b)

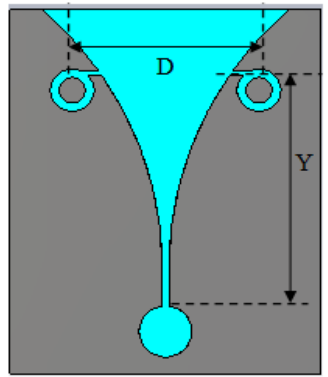

(d)
Fig. 7. Antenna Configuration with different Positions of the Printed Resonator. 


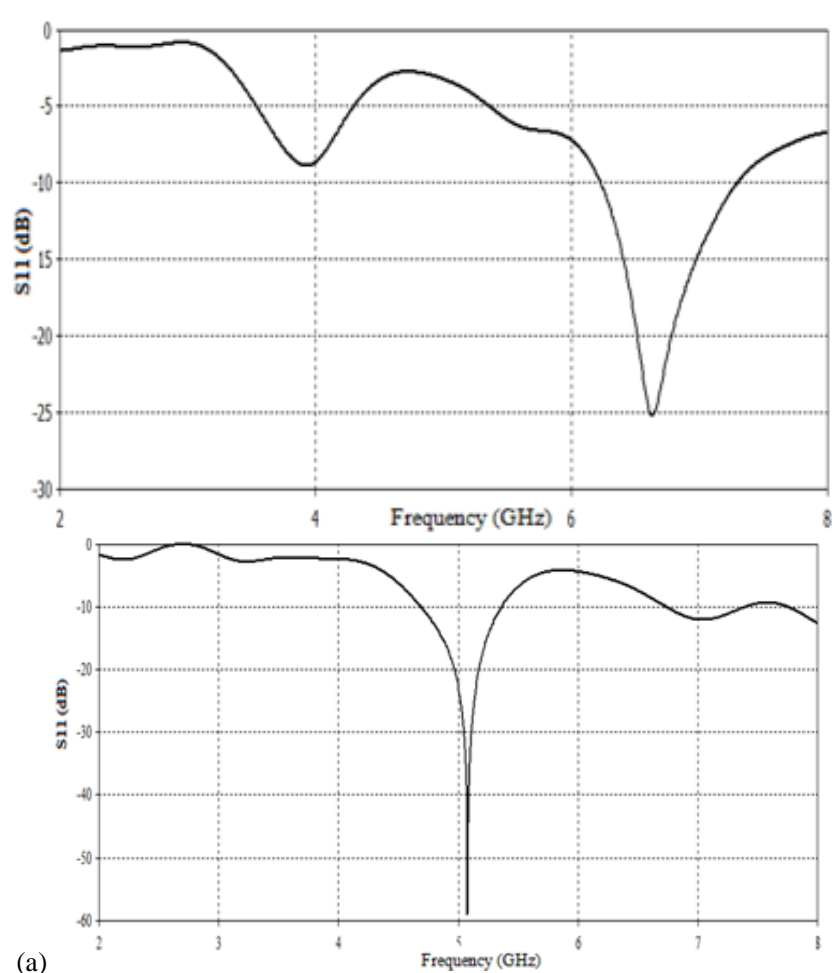

(b)

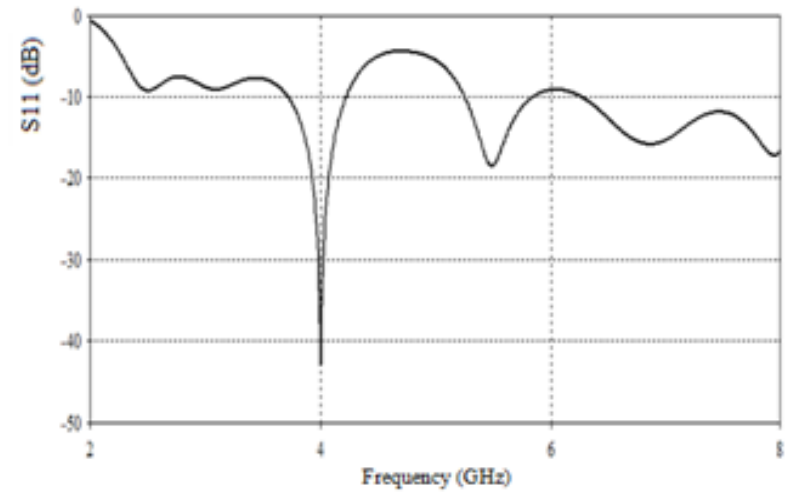

(c)

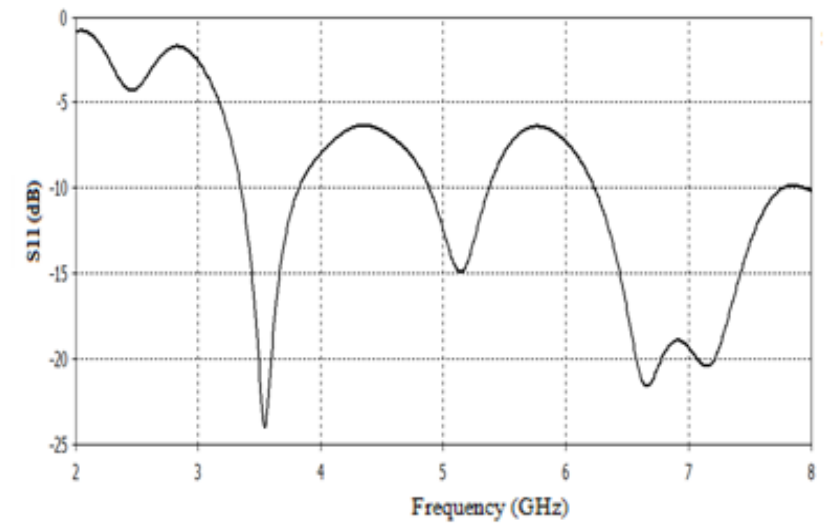

(d)

Fig. 8. Simulated Reflection Coefficients of four Configuration with different Positions of the Printed Resonator.
First, the level of the perturbation is about $16.5 \mathrm{~mm}$ and the current path length (D) is about $24 \mathrm{~mm}$ which practically corresponds to the half wavelength of $6.5 \mathrm{GHz}$ resonance frequency. So, a narrow band at $6.5 \mathrm{GHz}$ is obtained. Second, the elevation ( $\mathrm{Y}$ ) of the perturbation is increased, it is approximately $29 \mathrm{~mm}$ and the current path length (D) is 27 $\mathrm{mm}$ which means to the half wavelength of $5.2 \mathrm{GHz}$. Fig. 8(b) shows the return loss of this configuration which presents a narrow bandwidth at $5.15 \mathrm{GHz}$. Third, in Fig. 8(c) two narrow bands are obtained at $4 \mathrm{GHz}$ and $5.8 \mathrm{GHz}$ where the perturbation level is about $47.5 \mathrm{~mm}$ and $\mathrm{D}$ is about $38 \mathrm{~mm}$ (approximately equal to the half wavelength of $4 \mathrm{GHz}$ ). Finally, the level (Y) of the perturbation is increased as shown in Fig. 7(d); it is about $53 \mathrm{~mm}$ where the width (D) is $41 \mathrm{~mm}$ which is equal to the half wavelength of $3.5 \mathrm{GHz}$ resonance frequency. This configuration can offer three bands; two narrow bands at $3.5 \mathrm{GHz}$ and $5.2 \mathrm{GHz}$ and wideband from 6.2 $\mathrm{GHz}$ to $7.78 \mathrm{GHz}$. It can be concluded that increasing the level of disturbance enlarge the radiating region of the Vivaldi antenna which results the radiation of the antenna at lower frequencies and its matching at wide bandwidths. As well, this method can be used to properly control the frequency operating of the antenna which facilitates its command and agility.

\section{Four Modes Reconfigurable Vivaldi ANTENNA}

To demonstrate the functionality of the antenna to reconfigurate between the four modes previously explained, the four perturbations will be printed on the antenna and switched using PIN diodes as shown in Fig. 9(a). The frequency reconfigurability of the antenna is achieved by setting in the OFF state of the diodes that command one perturbation and in the ON state the other diodes which command the opening of the other perturbations. In this way, one perturbation will be activated which can force the disruption of the surface current and stop its circulation up, this allows the function of the antenna in one mode. Fig. 9(b) compares the different reflection coefficients of the four switched modes where sex narrow and agile bands are obtained. The first mode (M1) takes place when the four diodes (D1, D2, D3 and D4), which control the first perturbation, are in the $\mathrm{ON}$ state. In this case, one narrow band is obtained at $6.25 \mathrm{GHz}$. In the second mode (M2) which is attained by switching only the diodes D5 and D6 in the ON state, one narrow band is obtained that resonates at $5.2 \mathrm{GHz}$. Once the diodes D7 and D8 are activated, the third mode (M3) occurs which can present two narrow bands at $3.97 \mathrm{GHz}$ and $5.5 \mathrm{GHz}$. At last, the fourth mode (M4) is obtained when the superior perturbation is opened by the deactivation of diodes D9 and D10. This mode can offer two narrow bands at 3.5 $\mathrm{GHz}$ and $5.8 \mathrm{GHz}$. Because of the effect of PIN diodes, it can be observed that activation of the different modes using active switches has slightly affected the frequency response of the antenna. For example, activation of the fourth mode (M4) has result two narrow bands at $3.5 \mathrm{GHz}$ and $5.8 \mathrm{GHz}$ which is different to the result obtained in the precedent section with the passive configuration in mode M4. 


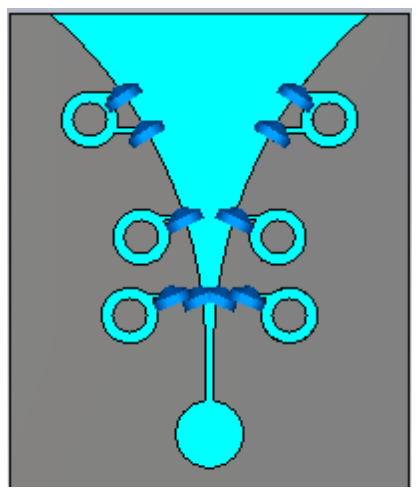

(a)

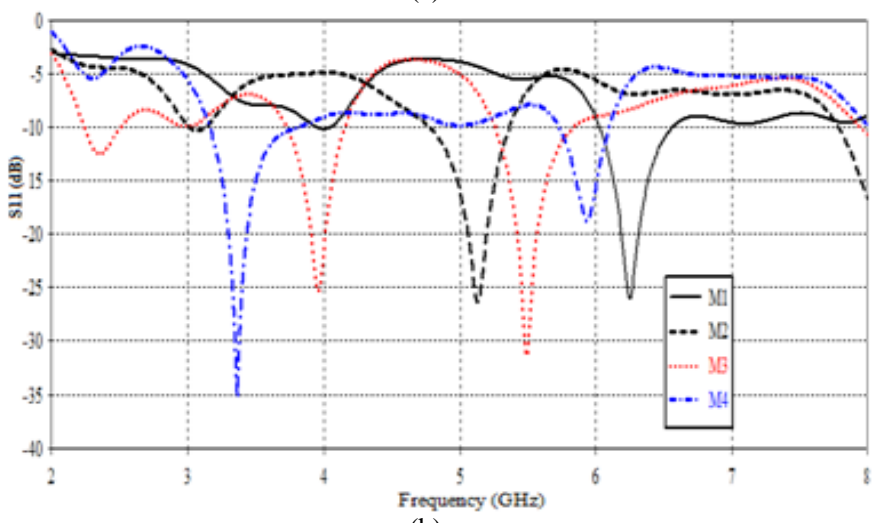

(b)

Fig. 9. Reconfigurable Vivaldi Antenna (a) and Reflection Coefficients or different Switching Modes (b).

To avoid the complexity of the bias circuit for PIN diodes and its effects in the antenna performances, four passive configurations of the defined modes are prototyped. Fig. 10 presents the designed and fabricated prototype for the different modes using ideal switches where the ON state of the PIN diode is modeled by a short-circuit while the OFF state is modeled by an open circuit. The surface current distributions excited at the resonance frequencies of the different modes using ideal switches are shown in Fig. 11. For the different cases, it is noticeably observed that the surface current distribution is more concentered at the active ring slot. Moreover, the active perturbation can stop the repartition of the surface currents on the upper part of the antenna which force the resonance at the desired frequency.

The simulated and measured reflection coefficients of the four switched modes are compared in Fig. 12. A good agreement between simulation and measured results is achieved, accepting slight difference is observed.

Fig. 13 plots an example of prototype antenna in the anechoic chamber, while Fig. 14 illustrates the simulated and measured radiation patterns in the $\mathrm{E}$ and $\mathrm{H}$-planes at the different frequencies obtained throughout the four switching modes. Generally, the measured results are in conformity with the simulated ones, except for a little decrease of the measured radiation patterns as compared to the simulated results. For the different operating modes, almost constant radiation patterns are obtained with maximum gain between $7 \mathrm{~dB}$ and $8 \mathrm{~dB}$ for the different operating frequencies in the direction of $0^{\circ}$ is obtained. It can be observed that the proposed configuration has not greatly affected the radiation patterns of the antenna. Table I summarizes the simulated and measured radiation performances which are obtained through the different modes of switching.
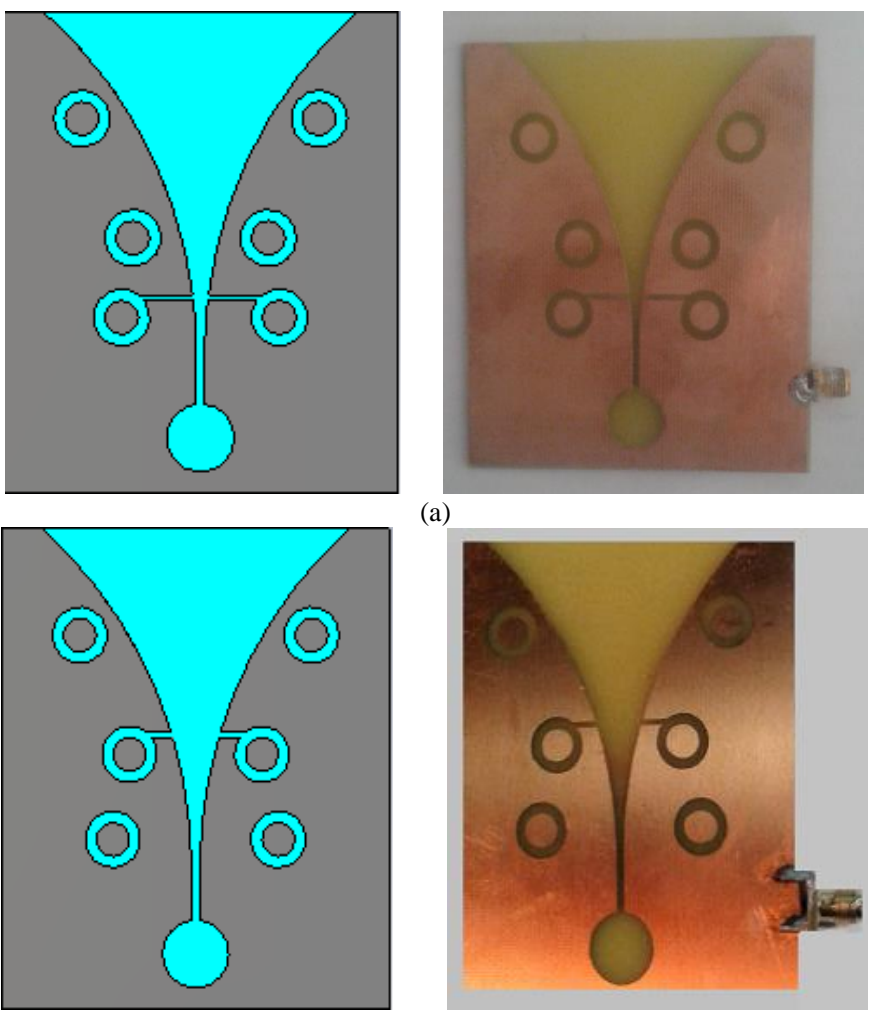

(a)

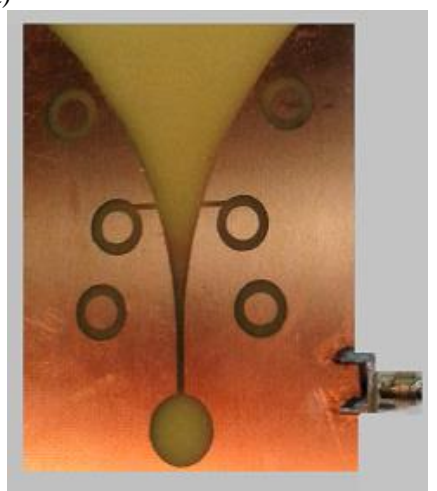

(b)
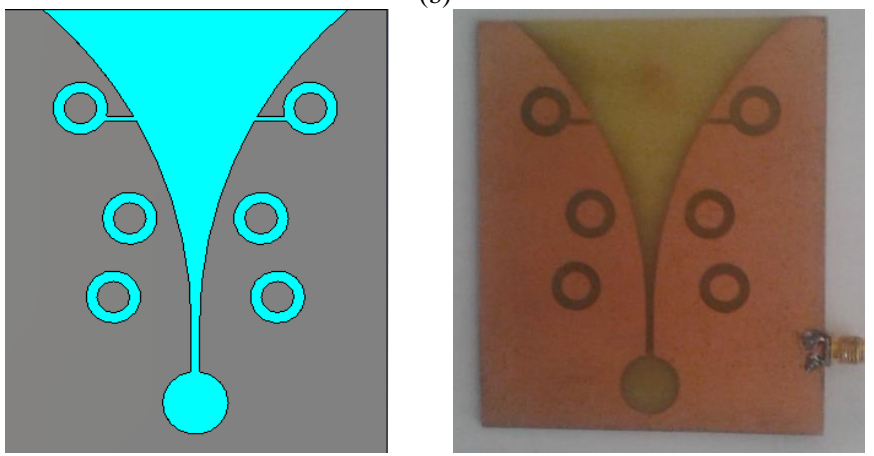

(c)
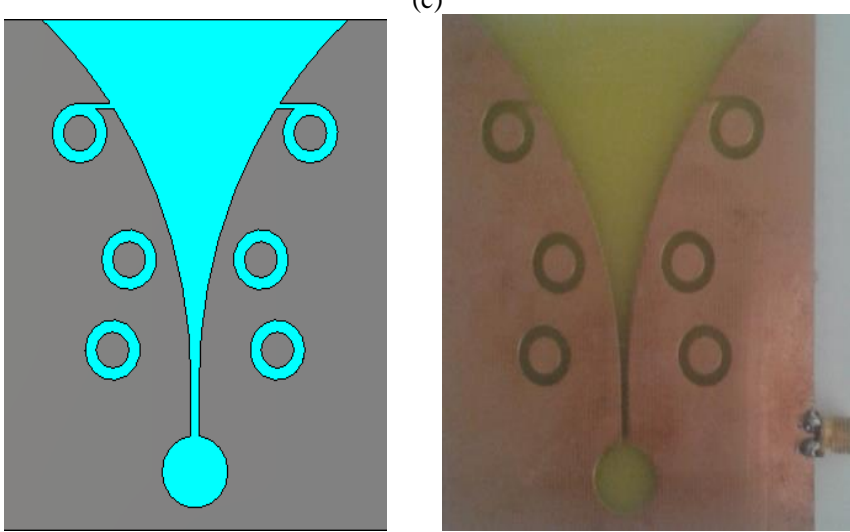

(d)

Fig. 10. Designed and Fabricated Prototype for different Modes: (A) Mode M1, (B) Mode M2, (C) Mode M3, (D) Mode M4. 


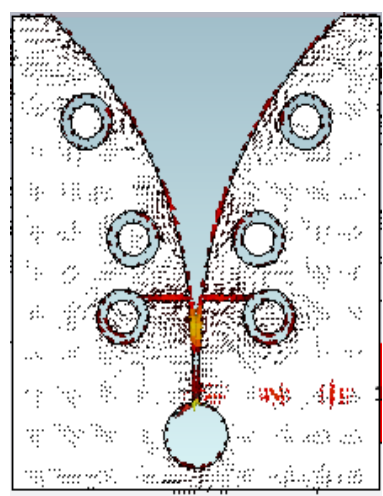

(a)

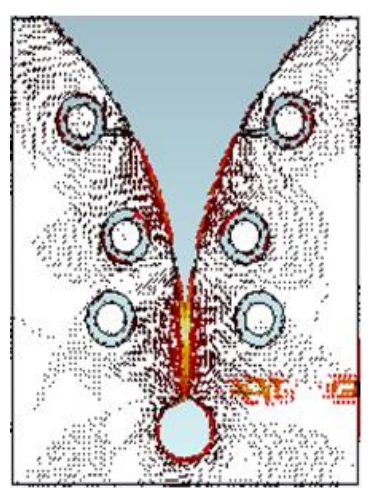

(c)

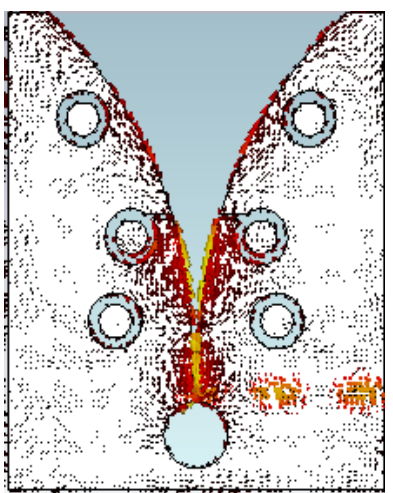

(b)

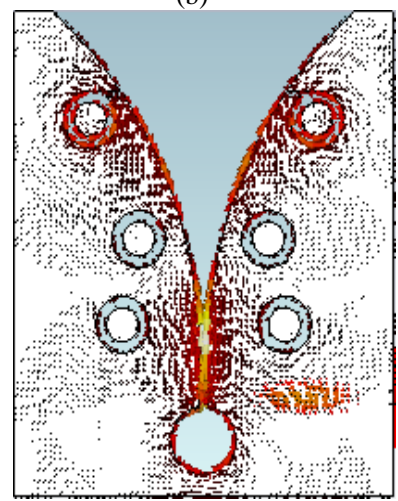

(d)

Fig. 11. Surface Currents Distribution for: (a) Mode M1 Excited at $6.5 \mathrm{GHz}$, (b) Mode M2 Excited at $5.2 \mathrm{GHz}$, (c) Mode M3 Excited at $4 \mathrm{GHz}$, (d) Mode M4 Excited at $3.5 \mathrm{GHz}$.

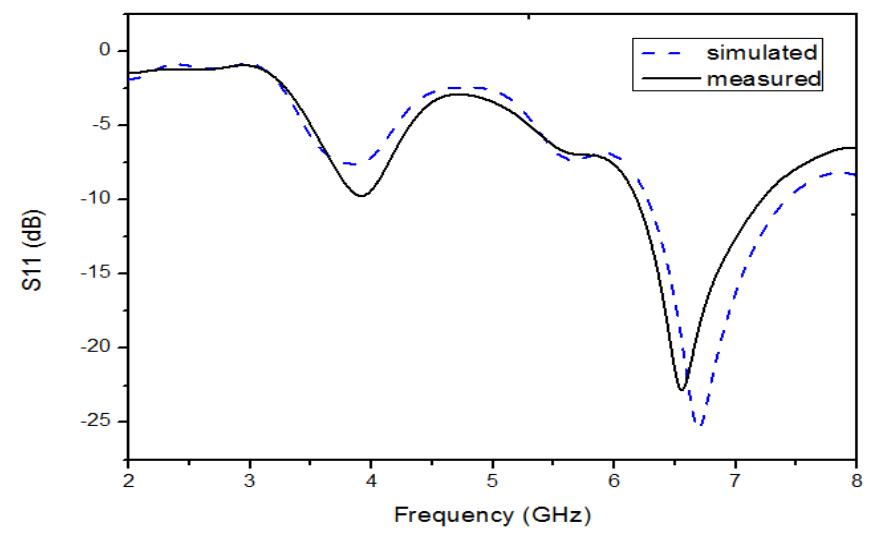

(a)

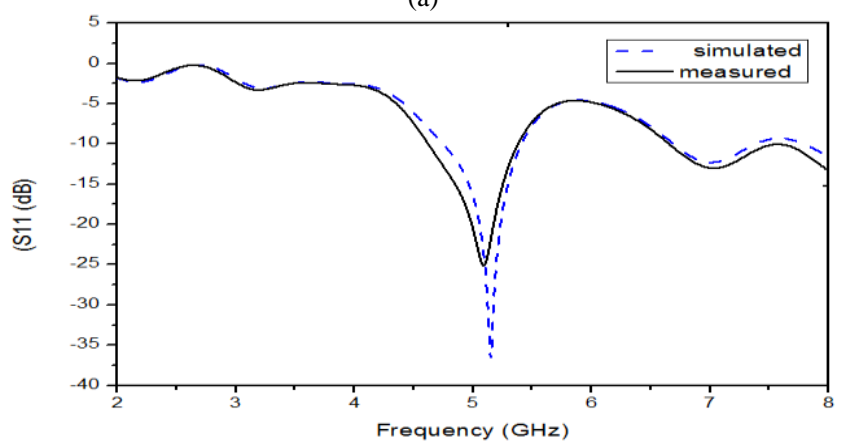

(b)

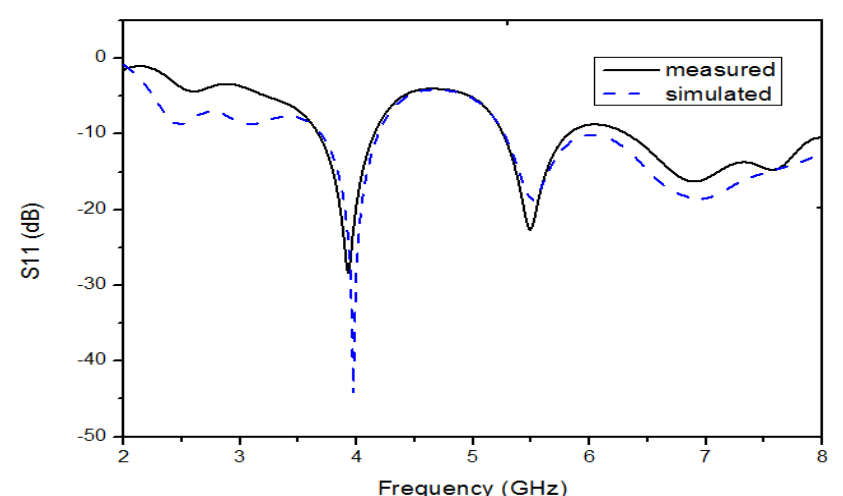

(c)

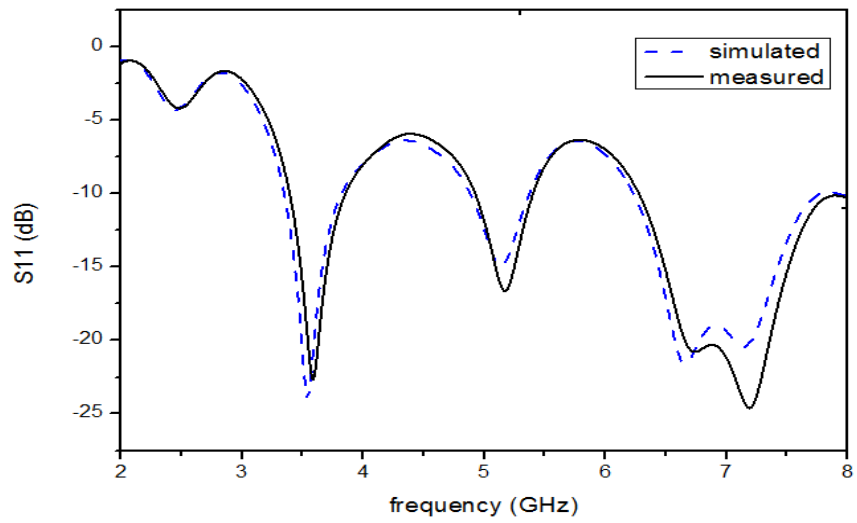

(d)

Fig. 12. Measured and Simulated Reflection Coefficients of different Operating Modes: (a) Mode M1, (b) Mode M2, (c) Mode M3, (d) Mode M4.

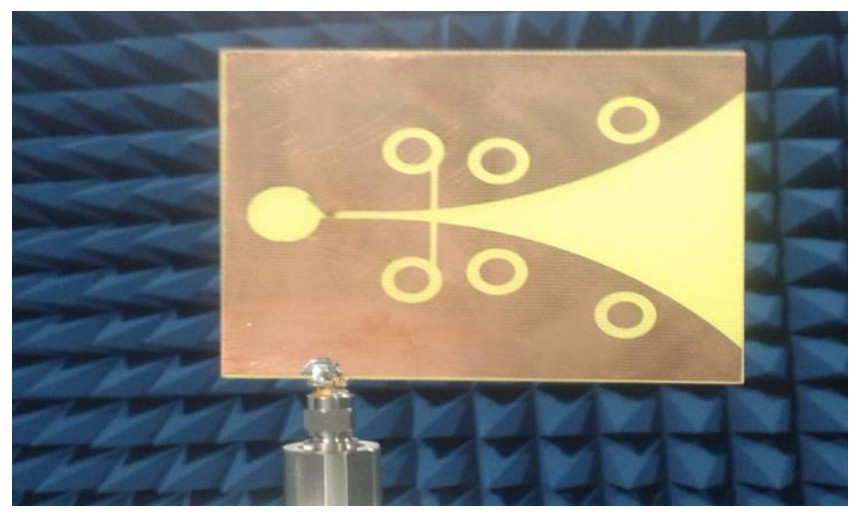

Fig. 13. Example of Prototype Antenna in Anechoic Chamber.
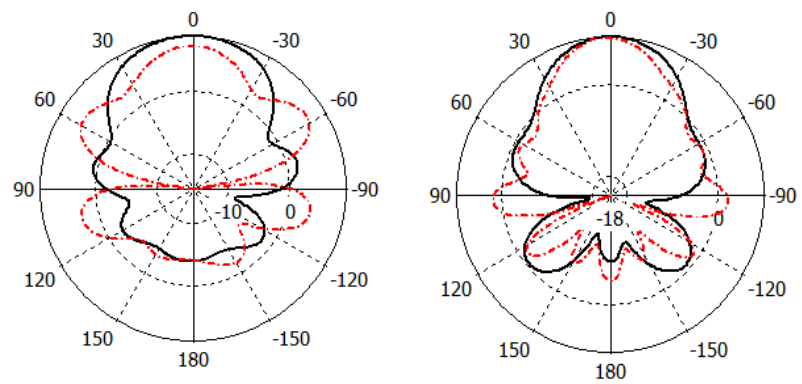

(a) 

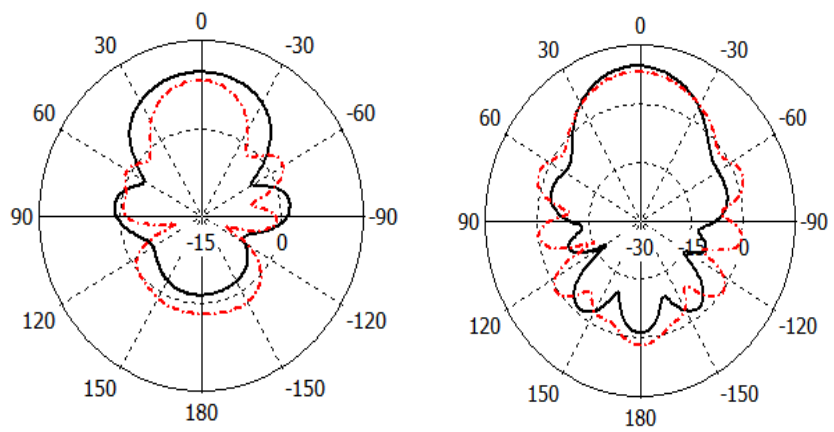

(b)
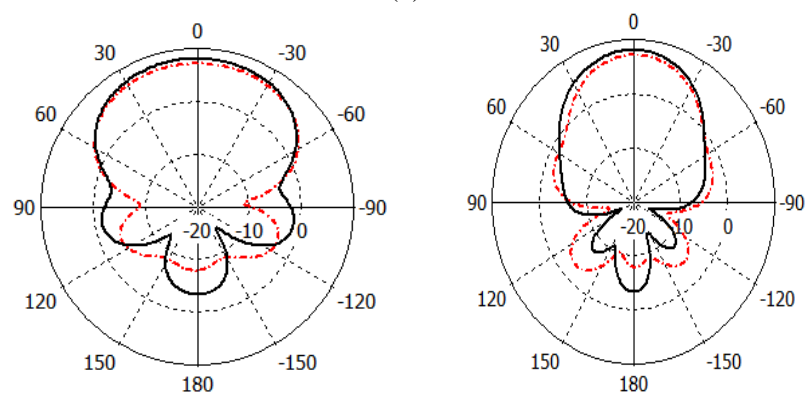

(c)
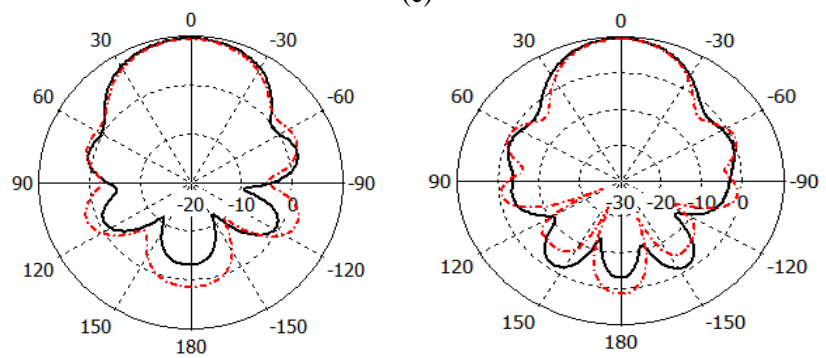

(d)
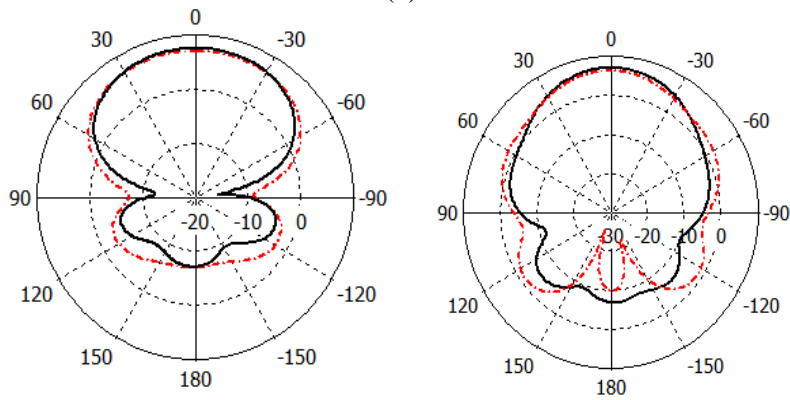

(e)
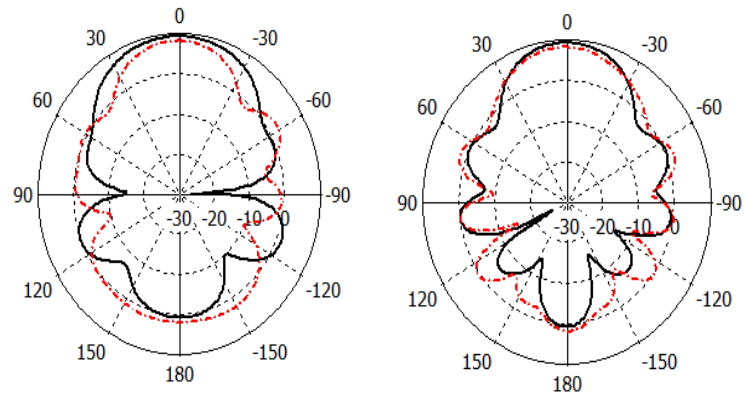

(f)

Fig. 14. Radiation Patterns in the E-Plane (Left) and H-Plane (Right) at (a) $6.5 \mathrm{GHz}$, (b) $5.2 \mathrm{GHz}$, (c) $4 \mathrm{GHz}$, (d) $5.5 \mathrm{GHz}$, (e) $3.5 \mathrm{GHz}$, and (f) $7 \mathrm{GHz}$.
TABLE I. RADIATION PERFORMANCES OF THE VIVALDI ANTENNA FOR DIFFERENT SWITCHING MODES

\begin{tabular}{|l|l|l|l|}
\hline Mode & $\begin{array}{l}\text { Resonance } \\
\text { frequency }(\text { GHz })\end{array}$ & $\begin{array}{l}\text { Simulated } \\
\text { Gain }(\text { dB })\end{array}$ & $\begin{array}{l}\text { Measured } \\
\text { Gain }(\text { dB })\end{array}$ \\
\hline Mode M1 & 6.5 & 8.07 & 7.53 \\
\hline Mode M2 & 5.2 & 9.54 & 8.41 \\
\hline Mode M3 & 4 & 9.53 & 7.22 \\
& 5.5 & 9.48 & 9.36 \\
\hline Mode M4 & $\begin{array}{l}3.5 \\
7\end{array}$ & 7.53 & 7.05 \\
& 9.26 & 8.15 \\
\hline
\end{tabular}

\section{CONCLUSION}

A frequency reconfigurable Vivaldi antenna with switched band pass resonator has been proposed. The Vivaldi antenna, which exhibits wideband of operation from $2 \mathrm{GHz}$ to $8 \mathrm{GHz}$, can be reconfigured by perturbing the surface current disruption. So, four switched perturbations are printed on the antenna metallization which can successfully control the surface current flow. Four switching modes are obtained that can offer several narrow bands at $3.5 \mathrm{GHz}, 4 \mathrm{GHz}, 5.2 \mathrm{GHz}$, $.5 .5 \mathrm{GHz}, 6.5 \mathrm{GHz}$ with satisfactory radiation patterns. In addition, measured and simulation results are in good agreement. Certainly, wideband multi-narrow bands reconfigurable antenna is extremely practical in more recent wireless applications that require dynamic agile frequency such as cognitive radio.

\section{REFERENCES}

[1] Rabiaa HERZI, Hsan ZAIRI, Ali GHARSALLAH: Reconfigurable Vivaldi Antenna with improved gain for UWB Applications. Microwave and Optical Technology Letters/ Vol. 58, No. 2, February 2016 DOI $10.1002 / \mathrm{mop}$.

[2] M. Bouslama, A.Gharsallah, M. Traii , and T. A. Denidni: BeamSwitching Antenna with a New Reconfigurable Frequency Selective Surface, IEEE Antennas and Wireless Propagation Letters 15, p 1159$1162,2016$.

[3] A. K. Singh, R.K. Gangwar, B. K. Kanaujia: Wideband and compact slot loaded annular ring microstrip antenna using L-probe proximityfeed for wireless communications. International Journal of Microwave and Wireless Technologies. 2015 doi:10.1017/S1759078715000446.

[4] Ullah S, Ahmad S, Khan BA, Flint JA (2018). A multi-band switchable antenna for Wi-Fi, 3G Advanced, WiMAX, and WLAN wireless applications. International Journal of Microwave and Wireless Technologies 1-7. https://doi.org/10.1017/ S1759078718000776.

[5] S. sharma and C. charu tripathi: Wideband to concurrent tri-band frequency reconfigurable microstrip patch antenna for wireless communication. International Journal of Microwave and Wireless Technologies, 1-8. 2016 doi:10.1017/S1759078716000763.

[6] Cai, Y., Y. J. Guo, and T. S. Bird: A frequency reconfigurable printed Yagi-Uda dipole antenna for cognitive radio applications. IEEE Transactions on Antennas and Propagation, Vol. 60, No. 6, 2905-2912, Jun. 2012.

[7] Kim JY, Ha SJ, Kim D, Lee B, Jung CW: Reconfigurable beam steering antenna using U-slot fabric patch for wrist-wearable applications. J. Electromagn. Waves Appl. 2012; 26: 1545-1553.

[8] C. Yong Rhee, and all. Frequency-reconfigurable antenna for broadband airborne applications. IEEE Antennas and Wireless Propag. Lett. 13 (2014).

[9] YingsongLi, Wenxing $\mathrm{Li}$, andQiubo Ye: ,A reconfigurable wide slot antenna integrated with sirs for UWB/multiband communication applications, Microwave and Optical Technology Letters Volume 55, Issue 1, pages 52-55, January 2013. 
[10] Yingsong Li, Wenxing Li, andQiubo Ye: A compact circular slot UWB antenna with multimode reconfigurable band-notched characteristics using resonator and switch techniques, Microwave and Optical Technology Letters Volume 56, Issue 3, pages 570-574, March 2014.

[11] N. Ojaroudi, Y. Ojaroudi, S. Ojaroudi: Compact Ultra-Wideband Monopole Antenna with Enhanced Bandwidth and Dual Band-Stop Properties. International Journal of RF and Microwave Computer-Aided Engineering Vol. 25, No. 4, May 2015.

[12] Kalteh, A. A., G. R. DadashZadeh, M. Naser-Moghadasi, and B. S. Virdee: Ultra-wideband circular slot antenna with reconfigurable notch band function, IET Microwaves, Antennas \& Propagation, Vol. 6, No. 1, 108-112, 2012.

[13] Y. Tawk and C. G. Christodoulou, Member, IEEE: A New Reconfigurable Antenna Design for Cognitive Radio, IEEE Antennas and Wireless Propagation Lettres, VOL. 8, 2009.

[14] MertKarahan, Demest S. Armagan Sahinkaya: A Reduced Size Antipodal Vivaldi Antenna Design for Wideband Applications, 2014 IEEE.
[15] Rabiaa HERZI, RamziGharbi, Hsan ZAIRI, Ali GHARSALLAH: A Tuneable Antipodal Vivaldi Antenna for UWB applications, 10th International Multi-Conference on Systems, Signals, and Devices (SSD13), Hammamet, Tunisia, March 2013.

[16] M. R. Hamid, P. Gardner, P. S. Hall, and F. Ghanem: Switched-band Vivaldi antenna, IEEE Trans. Antennas Propagat., vol. 59, no. 5, pp.1472-1480, May 2011.

[17] M. R. Hamid, P. Gardner, P. S. Hall, and F. Ghanem: Vivaldi Antenna with Integrated Switchable Band Pass Resonator, IEEE Trans. On Antennas and Propagation, Vol. 59, No. 11, November 2011.

[18] T.L. Yim, S.K.A. Rahim and R. Dewan: Reconfigurable wideband and narrowband tapered slot Vivaldi antenna with ring slot pairs. Journal of Electromagnetic Waves and Applications, 2013 Vol. 27, No. 3, 276287.

[19] F. Meng, S. K. Sharma, B. Babakhani: A Wideband Frequency Agile Fork-Shaped Microstrip Patch Antenna with Nearly Invariant Radiation Patterns. International Journal of RF and Microwave Computer-Aided Engineering/Vol. , No., 2016. 